\title{
Evaluating Paper Prototype for Tabletop Collaborative Game Applications
}

\author{
Marylia Gutierrez, Maurício Cirelli, Ricardo Nakamura, and Lucia Filgueiras \\ Escola Politécnica da Universidade de São Paulo, São Paulo, Brazil
}

\begin{abstract}
Identifying the natural gestures for a tabletop application is one of the most challenging tasks developers must accomplish in order to achieve a good system user interface. This problem is even more difficult in a collaborative environment. Cooperative Gestures allow richer interaction and must be evaluated when designing a new multi-user tabletop interface. In this paper we present the use of paper prototyping to analyze user interaction on a tabletop collaborative game application. Our results show that it is possible to extract natural gestures for an application using this technique, regardless of some limitations.
\end{abstract}

Keywords: Paper prototyping, game design, tabletop interfaces, test.

\section{Introduction}

The success of a digital game depends on several factors including technology, development process, risk management and marketing. Central among them is the design of the experience the game brings to players, which includes the game's interaction design.

For this reason, game development projects often include a pre-production phase that involves the creation of prototypes. Paper prototyping is a technique used to design and evaluate user interfaces. Its appeal comes from the flexibility, ease of changing the prototype and testing different scenarios. The simplicity makes users feel comfortable criticizing the system and besides being a lowfidelity prototype it leads to almost the same quantity and quality of critical user statements.

Multitouch interfaces allow cooperative gestures, which are single and combined commands executed from interactions made by more than one user [9]. One important concept when dealing with multitouch interfaces is the idea of groupware, which combines hardware and software to assist group interaction [10].

It is necessary to define an adequate set of gestures for the interface. Experiments such as the one by Hinrichs and Carpendale [1] show that gestures are not free of context, depending on the antecedent and subsequent gestures, as well as on the social context (e.g., user's age, sex). In the case of developing engaging games, there are several guidelines [2], but they do not explicitly take into account the special needs of every type of users. Tabletop multitouch interfaces allow the exploration of different types of applications [34. Games are 
suitable for experimenting with interface design methods because of the freedom in designing the tasks involved in their operation.

In this paper we present a experimental method and the results achieved testing on a common role-playing game (RPG) designed to be used with tabletop multitouch devices. We also discuss the feasibility of paper prototyping for the task of determining a set of most adequate gestures for multitouch-based digital games.

\subsection{Related Work}

In 6] we see two prototypes for a farm game tested with children which, after successive iterations, evolved into a product. Since the users were playing the game just for the fun, unexpected gestures were discovered.

Some insights regarding user behavior towards gesture definition can be found in [7. The study indicates that users rarely care about the number of fingers they employ, that one hand is preferred to two, among other results.

Other studies have shown that there are not significant differences between low fidelity and high fidelity prototypes. The choice of prototyping technique should be based on the group's composition and the desired focus [8].

\section{Paper Prototype}

Our prototype was tested using a RPG game. To create this game all the gestures for the activities needed to be chosen, which is not trivial, since it is not possible to know which gestures are the most natural and intuitive for the players. The paper prototype was developed to help in this task.

Another advantage of this early prototype is that some usability issues could be found and fixed before the development started. At last, after defining which gestures should be used, the gesture recognition system could be developed.

There are different types of prototypes, but in this case we needed a simple one that could represent the game satisfactorily. A paper prototype is simple to make and can be employed to analyze how the application is used.

In this prototype, an ordinary table was used with delimitations representing the screen; characters and life points were made out of paper as well. Cards representing the action areas were also created in paper so that the players could know the exact space which the skills could be performed. The prototype was then tested with four users and the results are presented in the next section. Before the test, the game was explained to the players, but none of the gestures were defined, leaving the players free to choose them.

\section{Results}

Different actions caused different reactions from the players. Gestures for movement were made naturally, which means the players did them intuitively, by 
dragging the characters to the place they wanted them to go. This gesture was made exactly the same way by all players. The simple attack/healing action was performed with small differences.

The special skills gestures required more thinking and discussion. When the gesture was individual, the player would stop for a while to think how to represent it and sometimes consult the other players. For collaborative gestures, there was always a time of discussion, where the players suggested gestures and voted to select one; sometimes the decision was no unanimous, which resulted in different gestures for the same action [1].

For the special skills, their names had a huge influence on the resulting gesture since the players were always trying to find gestures that would symbolize it. Therefore, if the exact same test was repeated with the same users but using different names for the special skills the resulting gestures could be totally different. Figure 1 shows the move gestures.
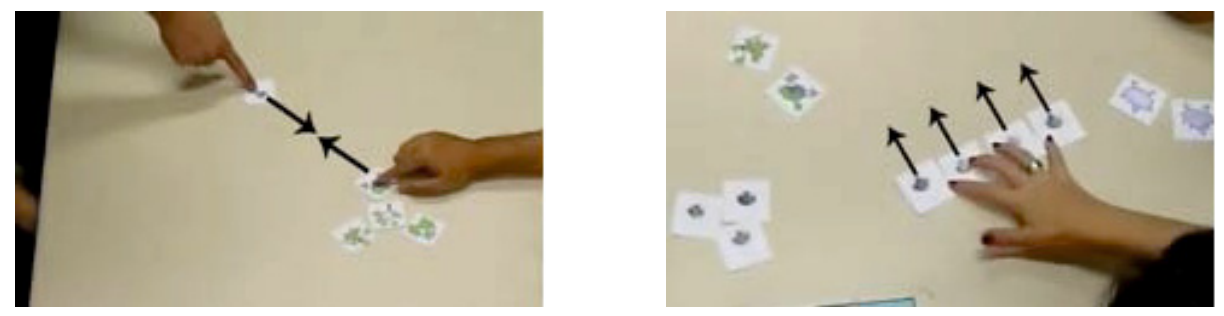

Fig. 1. Movement gestures

Despite being a successful approach in selecting gestures for actions, we have identified some limitations for tabletop applications design. The prototype was very similar to the final tabletop application, helping the players to imagine the game was real, but since it was not a real-time game, the speed and dynamics were compromised, creating a match that lasted far longer than on the real application.

In a real tabletop application, when the user touches the table, the targeted object is not necessarily going to move, but when we are working with paper, any touch would make the objects move and this inhibited the players. During the game players needed to make gestures that would not imply moving a character, and because they did not want to take the risk of moving them, the gestures were made above the "screen". This was a problem because sometimes the players made gestures in three dimensions, but our tabletop only works with $2 \mathrm{D}$ gestures. When this $3 \mathrm{D}$ gestures were made, the testers reminded the players that they were not acceptable gestures, making the players think even more on which gesture to do for the special skills, reducing the naturalness of some gestures.

\section{Conclusion}

Finding out which gestures are the most natural for an application is a challenging issue, and the paper prototype presented itself as one of the possible solutions 
to solve it. It has the ability to show some natural and intuitive gestures made by the players during the interaction.

The prototype helped not only at finding out these gestures, but also elements of the game that were missing or that were not clear to the players and other elements that were unnecessary. With the results of this test, changes could be made and the development of the game could start, avoiding a series of changes during or in the end of the development, when the game goes through tests with the users.

Further user studies still need to be performed in order to evaluate the relationship between skill names, social context, user's age and gender and the gestures performed.

\section{References}

1. Hinrichs, U., Carpendale, E.S.: Gestures in the wild: studying multi-touch gesture sequences on interactive tabletop exhibits, New York, NY, USA, pp. 3023-3032 (2011)

2. Malone, T.W., Lepper, M.R.: Making learning fun: A taxonomy of intrinsic motivations for learning. In: Snow, R.E., Farr, M.J. (eds.) Aptitude Learning and Instruction: III Conative and Affective Process Analyses, vol. 3, pp. 223-253. Lawrence Erlbaum Associates, Hilsdale (1987)

3. Kim, Y.-M.: Interactive Communion Tabletop- Graduate School of Advanced Imaging Science, Multimedia, and Film, Chung-Ang University, 221 Huksuk-Dong, Dongjak-Ku, 156-756, Seoul, Korea

4. Mori, T., Hamana, K., Feng, C., Hoshino, J.: Narrative Entertainment System with Tabletop Interface- 1-1-\#3M309, Tennodai 1-chome, Tsukuba-shi, Ibaraki, Japan

5. Bachl, S., Tomitsch, M., Wimmer, C., Grechenig, T.: Challenges for Designing the User Experience of Multi-touch Interfaces- 1 Research Group for Industrial Software (INSO), Vienna University of Technology, Austria; 2 Faculty of Architecture, Design and Planning, The University of Sydney, Australia

6. Marco, J., Baldassarri, S., Cerezo, E.: Bridging the Gap between Children and Tabletop Designers- Advanced Computer Graphics Group (GIGA) - Computer Science Department, Engineering Research Institute of Aragon (I3A) - University of Zaragoza, Spain

7. Wobbrock, J.O., Morris, M.R., Wilson, A.D.: User-Defined Gestures for Surface Computing - CHI 2009 Tabletop Gestures, Boston, MA, USA, April 7 (2009)

8. Johansson, M., Arvola, M.: A Case Study of How User Interface Sketches, Scenarios and Computer Prototypes Structure Stakeholder Meetings. In: Proceedings of HCI 2007 (2007)

9. Morris, M.R., Huang, A., Paepcke, A., Winograd, T.: Cooperative Gestures: MultiUser Gestural Interactions for Co-located Groupware. In: CHI 2006, Montréal, Québec, Canada, April 22-28 (2006)

10. Ellis, C., Wainer, J.: Groupware and Computer Supported Cooperative Work 